Revue d'histoire de l'enfance « irrégulière »

Le Temps de l'histoire

9 | 2007

Violences et jeunesse

\title{
La violence des jeunes dans les campagnes du Sud- Ouest au XIXème siècle : ethos agonistique et masculinité
}

\section{François Ploux}

\section{(2) OpenEdition \\ Journals}

Édition électronique

URL : http://journals.openedition.org/rhei/2072

DOI : 10.4000/rhei.2072

ISBN : 978-2-7535-1648-9

ISSN : 1777-540X

Éditeur

Presses universitaires de Rennes

Édition imprimée

Date de publication : 15 novembre 2007

Pagination : 79-89

ISSN : 1287-2431

Référence électronique

François PlouX, «La violence des jeunes dans les campagnes du Sud-Ouest au XIXème siècle : ethos agonistique et masculinité », Revue d'histoire de l'enfance « irrégulière » [En ligne], 9 | 2007, mis en ligne le 01 novembre 2009, consulté le 03 décembre 2020. URL : http://journals.openedition.org/rhei/2072 ; DOI : https://doi.org/10.4000/rhei.2072 


\section{La violence des jeunes}

\section{dans les campagnes du Sud-Ouest}

\section{au XIXème siècle :}

\section{ethos agonistique et masculinité}

Les historiens spécialistes du XIXème siècle ont beaucoup progressé, depuis une vingtaine d'années environ, dans la connaissance et dans la compréhension des violences paysannes. Un certain nombre de chercheurs, rompant délibérément avec une approche purement quantitative du crime, très en vogue dans les années 1960-1970 et qui était fondée sur l'assimilation de la violence à la déviance, se sont efforcés d'aborder cette violence comme un fait de culture, produit d'un ethos socialement construit et susceptible de faire l'objet d'une approche de type anthropologique.(2) La prise de conscience de l'existence d'un décalage persistant - il ne se résorbera progressivement qu'à partir des années 1860 - entre la norme juridique et la norme coutumière explique, au moins en partie, ce revirement épistémologique.

Il n'est plus question désormais de faire de la violence paysanne l'expression pure et simple de l'agressivité d'êtres frustes, impulsifs, irascibles, aux mœurs peu policées, cédant facilement aux émotions. Par un travail de contextualisation qui suppose un changement de focale (l'étude de cas est désormais privilégiée), il est possible de reconstituer l'environnement social, culturel, normatif du crime, et de saisir les intentions, les motivations probables de son auteur.

Cette nouvelle manière de lire l'archive judiciaire permet en particulier de mieux cerner l'association entre jeunesse et violence que révèlent les documents de l'époque.

\section{Des enfants précocement exposés à la violence}

Faute de prendre en considération l'expérience que l'enfant faisait de la violence à l'intérieur de la famille, par conséquent aux premiers stades de sa socialisation, il est difficile de comprendre la brutalité que pouvaient revêtir les relations sociales au sein des communautés villageoises. Sans aller jusqu’à

(1) Maître de confé-

rence à l'université

de Bretagne-Sud, Lorient.

Centre de recherches

historiques de l'Ouest

(CERHIO-FRE

CNRS 3004).

(2) Dans une synthèse récente, Jean-Claude Farcy propose un état des lieux de la recherche en histoire de la criminalité : L'histoire de la justice française de la Révolution à nos jours.

Trois décennies de recherches, Paris, PUF, 2001, 494 p. Voir encore : Frédéric Chauvaud, [dir.],

L'histoire contemporaine et les usages des archives judiciaires (I800-1939), Paris, H. Champion, 1998, 490 p. ; Frédéric Chauvaud, Jean-Luc Mayaud, [dir.], Les vio-

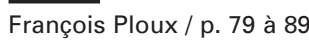


lences rurales au quotidien. Actes du zième colloque de l'Association des ruralistes français, Paris, La boutique de l'Histoire, 2005, 379 p., ainsi que le numéro spécial qu'Éthnologie française a consacré à la question des violences ("Violence, brutalité, barbarie ", 1991/3).

(3) Pierre Bourdieu, Méditations pascaliennes, Paris, Seuil, "Liber ", 1997 , p. 275.

(4) Voir notamment : Élisabeth Claverie, Pierre Lamaison, L'impossible mariage. Violence et parenté en Gévaudan, XVIIème-XVIIİ̀meXIXème siècles, Paris, Hachette, 1982, 361 p. ; Georges Augustins, Comment se perpétuer? Devenir des lignées et destins des patrimoines dans les paysanneries européennes, Nanterre, Société d'ethnologie, 1989, 434 p. ; Alain Collomp, " Conflits invoquer cette loi de conservation de la violence qu'énonce Pierre Bourdieu dans les Méditations pascaliennes, ${ }^{(3)}$ on peut faire l'hypothèse qu'une exposition précoce et continue à la violence (subie, ou tout simplement observée, vécue), conditionnait le rapport de l'adulte à la violence. Or, loin d'être le havre de paix que se plaisait à imaginer la sociologie leplaysienne, la famille-souche méridionale était travaillée par des conflits d'une très grande intensité : bon nombre s'achevaient dans les coups. Et les violences domestiques n'étaient pas seulement banales : ces affrontements entre proches parents, frappés d'interdit et par conséquent très peu ritualisés, étaient terriblement meurtriers. De manière générale, la brutalité des conflits était inversement proportionnelle à la distance sociale entre les adversaires aux prises. La rupture des solidarités élémentaires, de type familiales ou vicinales - on sait l'importance que pouvaient revêtir les rapports de voisin à voisin en pays d'habitat semi-dispersé-, débouchait fréquemment sur des conflits aux conséquences atroces.

Grâce aux nombreux travaux qu'historiens et anthropologues ont consacré à la famille paysanne traditionnelle, on saisit bien désormais la logique de la conflictualité domestique. (4) Dans le Midi de la France, où la " maison ", succession de chefs de famille établis sur un domaine stable, constituait la cellule élémentaire de toute l'organisation sociale, la transmission de l'intégralité du patrimoine foncier à un seul enfant était un véritable impératif : c'est en effet à cette condition que la maison, association d'une lignée et d'une exploitation, pouvait se perpétuer dans le temps. L'avenir de la maison dépendait de la réussite d'un certain nombre d'opérations qui constituaient autant de points d'articulation des stratégies de reproduction déployées par les familles. Ainsi fallait-il désigner, parmi les membres de la fratrie, le garçon appelé à prendre la suite de son père, lui transmettre le pouvoir domestique, négocier avec les cadets le montant de la « légitime » en numéraire dont le versement signifiait renonciation à une part en nature du patrimoine, marier le futur chef de maison à une cadette dotée...

Cependant, ce système de reproduction des maisons engendrait des tensions, des conflits et des violences. Si le principe de la nécessaire perpétuation de la maison était à ce point intériorisé par les agents sociaux que personne, pas même ceux qui en faisaient les frais - c'est-à-dire les cadets, condamnés au célibat, à l'exil ou à la domesticité -, ne venait contester une coutume dont le 
caractère inégalitaire garantissait l'inaliénabilité du patrimoine, tout n'allait pas pour autant sans tiraillements. L'analyse des antagonismes ayant donné lieu à des poursuites judiciaires, lesquels ne different des affrontements ordinaires que par leur intensité, permet d'observer la logique qui sous-tendait la conflictualité familiale. ${ }^{(5)}$ Les querelles mettant aux prises le mari à sa femme, le chef de maison à son fils aîné, ce dernier à ses cadets... avaient tous quelque chose à voir avec le processus de transmission du patrimoine et du pouvoir domestique. Le système coutumier lui-même pouvait générer des situations conflictuelles. On se contentera d'évoquer le cas - typique - des violences exercées par un gendre sur sa femme ou sur sa belle-famille. Cette configuration est en effet très révélatrice des rapports entre violence et masculinité. Dans les familles sans héritier mâle, l'une des filles, qui a donc le statut d'héritière, a épousé un cadet, " entré en gendre " dans la maison de sa femme. L'époux, dépourvu de l'autorité que confere le statut d'héritier, occupe en outre dans la maison la place d'une femme. D'où une situation extrêmement inconfortable, entraînant une véritable crise d'identité chez ceux qui, bien que de sexe masculin, ne détiennent pas la réalité du pouvoir dans la maison. La violence du gendre - elle est souvent terrible vient compenser une masculinité amputée.

Sans qu'il soit possible d'émettre sur ce point un avis définitif, il est fort probable que ces conflits internes à la maisonnée se sont multipliés et intensifiés au début du XIXème siècle. Le Code civil offrait désormais aux cadets en négociation avec leurs parents au sujet du montant de leur légitime un moyen de pression particulièrement efficace. L'autorité du père, rarement contestée, ne s'en trouvait pas moins diminuée par l'abolition théorique du système coutumier qui l'autorisait à attribuer ses biens à un seul de ses enfants. ${ }^{(6)}$ L'allongement de l'espérance de vie plaçait nombre de maisons sous l'autorité de parents âgés, ce qui non seulement fragilisait l'équilibre économique de l'exploitation, mais surtout avait le don d'exaspérer nombre d'aînés, impatients de régner en maîtres. Le contrôle des naissances multipliait les familles sans descendance mâle, et par voie de conséquence les mariages en gendre. En outre, du fait de l'emprise croissante de l'économie monétaire dans les campagnes, les jeunes paysans prenaient davantage conscience de la valeur marchande de leur travail, mais aussi de ce que pouvait coûter l'entretien des parents. Cette aptitude croissante au calcul explique que les enfants aient été familiaux et groupes de résidence en Haute-

Provence ", Annales ESC,

mai-juin 1981 , p. 408 -

425 ; Nicole Castan,

"Condition féminine et

violence conjugale dans la société méridionale française au XVIIIİ̀me siècle ", in Le modèle familial européen : normes, déviances, contrôle $d u$ pouvoir, Paris, De Boccard, 1986, p. 175 184. Pour le Quercy : François Ploux, Guerres paysannes en Quercy. Violences, conciliations et répression pénale dans les campagnes du Lot (I8IoI860), Paris, La boutique de l'Histoire, 2002, p. 19-54.

(5) Georges Augustins explique la violence caractéristique des sociétés " à maison " du sud de la France, où les conflits intra et inter familiaux étaient innombrables, par la difficulté qu'éprouvaient les maisons à concilier deux principes 


\begin{tabular}{|c|c|}
\hline la perpétuation de la & \\
\hline lignée, de la continuation & \\
\hline du nom patronymique & (8) Élisabeth Claverie, \\
\hline d'une part, celui de la & Pierre Lamaison, op. cit. \\
\hline perpétuation du patrimoi- & \\
\hline ne d'autre part. Georges & (9) Il n'est pas de \\
\hline Augustins, op. cit, p. 324- & meilleure analyse \\
\hline 326. Nous avons discuté & des stratégies matrimo- \\
\hline cette hypothèse dans & niales dans le système \\
\hline Guerres paysannes en & des stratégies de reproduc- \\
\hline Quercy, op. cit., p. 92-93. & tion que celle que \\
\hline & propose Pierre Bourdieu: \\
\hline (6) Sur les procédés mis & Le bal des célibataires. \\
\hline en œuvre pour contourner & Crise de la société paysanne \\
\hline la loi : Jean Hilaire, & en Béarn, Paris, Seuil, \\
\hline "Vivre sous l'empire du & « Points essais », 2002, \\
\hline Code civil. Les partages & $268 \mathrm{p}$. \\
\hline successoraux inégalitaires & \\
\hline au XIXème siècle ", & (10) Pensons encore \\
\hline Bibliothèque de l'École des & à ces petites filles violées \\
\hline chartes, t. 156,1998 , & que leurs parents cor- \\
\hline p. $117-141$. & rigeaient lorsqu'ils décou- \\
\hline & vraient l'affaire. François \\
\hline (7) Sylvie Lapalus, & Ploux, Guerres paysannes, \\
\hline La mort du vieux. Une & op. cit., p. 270, note 158. \\
\hline histoire du parricide & \\
\hline au XIXème siècle, Paris, & (11) Voir à ce propos \\
\hline Tallandier, 2004, particu- & les commentaires de \\
\hline lièrement p. 401-463. & Maurice Agulhon dans \\
\hline & son introduction aux \\
\hline & Mémoires de Léonard de \\
\hline & Martin Nadaud (Paris, \\
\hline & Hachette, 1976,557 p.) \\
\hline
\end{tabular}

moins spontanément disposés que par le passé à se sacrifier pour la maison. ${ }^{(7)}$

Il existait par ailleurs un lien structurel entre la conflictualité intrafamiliale et celle qui se déployait à l'intérieur de l'espace des échanges matrimoniaux. La mise en évidence de l'articulation entre ces deux niveaux de violence constitue l'un des principaux apports du travail qu'Élisabeth Claverie et Pierre Lamaison ont consacré, il y a maintenant plus de quinze ans, aux criminels du Gévaudan. ${ }^{(8)}$ Dans la mesure où le mariage de l'héritier, qui signifiait l'entrée d'une dot dans la maison et conditionnait l'avenir de la lignée, était une pièce maitresse dans la panoplie de stratégies de reproduction familiale, ${ }^{\left({ }^{9}\right)}$ une vive concurrence opposait les maisons de rang équivalent situées sur un même marché matrimonial. Nombre de violences observées au début du XIXème siècle (mais pas toutes, cela va de soi) étaient à mettre sur le compte de la tension qui régnait, dans les villages, du fait de cette intense compétition entre maisons rivales.

Le monde de l'enfance était par conséquent marqué du sceau de la violence. La violence était familière à l'enfant, qu'elle s'exerçât dans le cadre d'un conflit domestique ou dans les relations quotidiennes. Le châtiment corporel était par exemple un mode habituel d'exercice de l'autorité parentale. ${ }^{(10)}$ Les chefs d'exploitation usaient du même procédé à l'égard des petits domestiques employés comme bergers ou comme servantes. La violence imprégnait jusquaux jeux des enfants. ${ }^{(11)}$ On comprend mieux, dans ces conditions, la propension des paysans devenus adultes à user de violences pour régler leurs querelles.

\section{Une culture de la violence}

La fragilisation du système de reproduction des maisons consécutive aux mutations démographiques et ins- 
titutionnelles post-révolutionnaires ne peut à elle seule expliquer la brutalité des mœurs paysannes. Dans cette société, la violence, celle des jeunes en particulier, était l'expression d'une culture, la manifestation d'une éthique particulière tout autant qu'un symptôme d'anomie. Une conception de l'honneur valorisant l'agression caractérisait les sociétés villageoises du Midi, et sans doute, à des degrés variables, l'ensemble de la paysannerie française, avant que les institutions englobantes - École, Église, Justice... - ne parviennent, par un travail de sape, à disqualifier les conduites violentes. Aux alentours des années 1850, on pouvait encore observer, en particulier dans le sud du Massif central, cet " ethos agonistique " propre aux cultures d'honneur, que toute une littérature anthropologique consacrée au monde méditerranéen n'a cessé d'explorer. Dans le Gévaudan ou le Quercy, l'honneur était une composante fondamentale du statut. Se comporter en homme d'honneur signifiait non seulement être capable de relever la moindre provocation, de laver tous les affronts, même les moins évidents, mais encore nécessitait de faire la démonstration d'une certaine agressivité à l'égard du monde environnant. La bravade, la morgue, le défi, mais aussi la violence, étaient autant d'expressions de la puissance sociale. Par conséquent, cette propension au défi était proportionnée au rang occupé dans la hiérarchie locale : pareille attitude caractérisait d'abord les grandes maisons, capables, grâce à leur influence et à leurs relations, d'échapper aux sanctions judiciaires que le comportement belliqueux de leurs membres aurait dû théoriquement entraîner.

Cependant, dans cette société, on observait bien peu de conflits d'honneur " purs ", affrontements où la défense du point d'honneur aurait été l'unique motivation des adversaires aux prises. En effet, le moindre différend était perçu comme mettant en jeu la dignité des protagonistes. De là, la propension à convertir les antagonismes les plus divers (concurrence sur le marché matrimonial, rivalité amoureuse, querelle de voisinage, compétition autour du pouvoir local...) en affaire d'honneur. Comme, par ailleurs, la violence physique était considérée comme un mode normal, légitime, de réparation de l'honneur outragé, on comprend la fréquence des actes de vengeance, qui s'intégraient parfois à de véritable cycles.

Toutefois, cette culture agonistique ne se traduisait pas exclusivement par des attentats mûrement réfléchis. Les cycles vindicatoires opposant des maisons rivales étaient somme toute assez exceptionnels. ${ }^{(12)}$ La rixe de cabaret
(12) Il faut être conscient de l'effet de grossissement qu'induit l'usage quasi-exclusif de l'archive judiciaire. Il n'en reste pas moins que ces documents, utilisés dans la perspective d'une étude du conflit, et pas nécessairement de la déviance, constituent une source de toute première importance pour qui s'attache à l'étude anthropologique des sociétés du passé. 
(13) Sur le rapport entre violence et agressivité : Marshall Sahlins, Critique de la sociobiologie. Aspects anthropologiques, Paris, Gallimard, 1980, 193 p.

(14) On s'y retrouvait pour arranger une affaire, acquitter une dette, négocier une transaction. Il va sans dire que l'auberge était avant tout un lieu de convivialité et de divertissement : on y buvait, on y dansait, on y jouait, on y discutait abondamment..

\section{(15) Le métier d'édu-} cateur. Les instituteurs de I9oo, les éducateurs spécialisés de 1968, Paris, Éditions de Minuit, 1983, p. 110. Sur l'identité masculine de l'homme des champs, voir André Rauch, Crise de l'identité masculine. 1789-19I4, Paris, Hachette, «Pluriel ", 2000, p. 121-176. était en revanche une forme des plus banales de violence d'honneur. La fréquentation du cabaret était presque exclusivement le fait des hommes, en particulier des hommes jeunes. Ils s'y retrouvaient soit le dimanche soir, soit à l'occasion d'une foire ou d'une fête. Les combats au couteau y étaient fréquents et d'une très grande brutalité. Cependant il ne faudrait pas interpréter ces déchaînements de violence comme autant de symptômes d'un relâchement du contrôle des affects qu'occasionnerait une trop abondante consommation de vin. Analyser cette violence en termes de pulsions agressives serait réducteur. ${ }^{(13)}$ C'est au cabaret que s'exprimait librement, dans la violence des coups de bâton ou de couteau, et alors que les paysans échappaient à la surveillance du maire et des gendarmes, ce culte de la force physique qui caractérisait la conception masculine de l'honneur. Le cabaret était en effet le lieu par excellence du règlement de compte (aux deux sens du terme), ${ }^{(14)}$ mais aussi du défi et de l'exhibition de la force, de la démonstration du courage. D'où la tension qui régnait dans les débits de boissons aux heures d'affluence : ignorer une offense (laquelle pouvait se manifester par une allusion, une parole à double sens, un geste), c'était prendre le risque de perdre la face en public, de voir sa réputation ternie par les commentaires que l'incident ne pouvait manquer de susciter dans le village.

\section{Violence et socialisation : la formation d'un ethos viril}

C'est à l'adolescence, au moment même où ils cessaient de se mêler aux filles pour désormais les "fréquenter ", c'est-à-dire les courtiser de manière très codifiée et sous la surveillance du groupe local ou de ses mandataires, que les garçons apprenaient à se conformer à cette éthique agressive qui fondait leur identité d'hommes, les distinguait des femmes dont on exigeait, au contraire, retenue et pudeur. Le travail sur l'exploitation fournissait l'occasion de cet apprentissage, qui s'accomplissait en même temps que s'opérait une progressive différenciation des tâches. Une atmosphère d'émulation virile régnait au sein de la domesticité. Elle était au cour du travail en commun, sur les grands domaines comme sur les petites exploitations familiales. Le labeur prenait des allures de véritable mise à l'épreuve pour le jeune paysan. Les ouvriers expérimentés abreuvaient de leurs sarcasmes la nouvelle recrue frêle et maladroite. Faire la démonstration de sa force revenait au contraire à 
prouver sa virilité. Dans la société paysanne, écrit Francine Muel-Dreyfus, "l'apprentissage sous le regard des hommes est porté par un code d'honneur qui oblige à toujours aller à la limite de ses forces ». ${ }^{(15)}$ L'attribution de nouvelles responsabilités, l'élévation dans la hiérarchie des valets de ferme s'accompagnaient de rites (par exemple le don d'un couteau ou d'un bâton, autant de marqueurs de l'identité masculine) qui signalaient aussi bien la compétence acquise que la virilité. ${ }^{(16)}$

L'apprentissage des valeurs qui fondaient la masculinité s'effectuait aussi dans le cadre de la sociabilité juvénile. L'incorporation de l'adolescent au groupe de jeunesse constituait donc à cet égard une étape des plus importantes. Elle revêtait une dimension quasi initiatique, quand bien même cette institution était généralement dépourvue d'organisation formelle. ${ }^{(17)}$ Le rituel d'intégration consistait à marquer la rupture entre le monde de l'enfance et l'univers grivois de la jeunesse. Il faut citer à ce propos le témoignage de l'instituteur Baptiste Sandre, né en 1824, qui grandit à Chantemerle, dans le Châtillonais. La scène se déroule au tout début des années 1840 :

" Il existait à Chantemerle une société de jeunesse. Pour y être reçu, deux conditions et un devoir étaient de rigueur. La lère était d'avoir 17 ans ; la 2ème de payer à boire, le jour de la réception. Quant au devoir, il consistait à pourchasser tout enfant qui s'approchait des membres de la société pour écouter leur conversation. Cette précaution était louable. Car parfois il aurait peut-être pu entendre quelque chose de peu édifiant. La réception se faisait à l'auberge, en présence de tous les membres. On allumait quelques chandelles en guise de cierges, et l'on exécutait des chants appropriés à la circonstance [L'auteur évoque plus loin "le refrain d'une chanson guerrière ou bachique”. " (18)

La licence et les démonstrations de virilité (cracher, péter, roter...) ${ }^{(19)}$ étaient au fondement de la sociabilité juvénile. Par ailleurs, les interventions de la jeunesse dans la vie du village contribuaient à reproduire tout un système de représentation de la masculinité et de la féminité : c’étaient les célibataires, on le sait, qui étaient chargés de sanctionner les maris battus, dominés ou trompés, les femmes soupçonnées de prendre l'initiative sur le plan sexuel, les homosexuels, les filles réputées faciles, etc. On connait le répertoire de la sanction : promenade sur l'âne (l' " assouade »), charivari, jugement de carnaval, mai injurieux, chansons satiriques...
(16) Jean-Claude Farcy,

La jeunesse rurale dans

la France du XIXème siècle, Paris, éditions Christian, 2004, p. 57-58.

(17) La perception de droits d'entrée ou l'existence de dignitaires élus (abbés, capitaines, chefs de la jeunesse) ne sont avérées que dans quelques provinces (Jean-Claude Farcy, La jeunesse rurale, op. cit., p. 148-149).

(18) La classe ininterrompue. Cahiers de la famille Sandre, enseignants. 1780-1960, présenté par Mona Ozouf, Paris, Hachette, 1979, p. 187-188.

(19) André Rauch, op. cit. 
(20) Pour le Quercy, François Ploux, op. cit., p. 174.

(21) M. Diverrès, "La faucherie du coq ", Bulletin de la Société archéologique du Finistère, 1887 , p. 75 et 327-330.

(22) Robert J. Bezucha, «The Moralization of Society : The Enemies of Popular Culture in the Nineteenth Century ", in Jacques Beuroy, Marc Bertrand, Edward T. Gargan, [ed.], Popular Culture in France. The Wolf and the Lamb: from the Old Regime to the Twentieth Century, Stanford, Anma Libri, 1977, p. 175-187.

(23) Pierre Joigneaux, Monographie de la commune de Ruffey-les-Beaune (Côte-d'Or), Paris,

Librairie veuve

Rougemont, 1888.

(24) Francine MuelDreyfus, op. cit., p. 108.
La violence, mise en évidence de la force musculaire et de la virilité, était une autre dimension constitutive de cette sociabilité des garçons célibataires. Les divertissements que la jeunesse villageoise était chargée d'organiser les jours de fête votive ou à la foire étaient souvent très brutaux. L'un des jeux les plus prisés consistait à décapiter ou à lapider une volaille. ${ }^{(20)} \mathrm{La}$ " faucherie " du coq était pratiquée en Basse-Bretagne au début XIXème siècle. Le juge de paix du deuxième canton de Lorient observe, en 1887, " que la principale réjouissance des écoliers, le jour de la fête de saint Nicolas, leur patron au Guéméné, consistait à couper le cou à des coqs suspendus par les pattes à des branches d'arbre. Ils attaquaient le pauvre volatile, le sabre au poing et les yeux bandés. Ce jeu n’a cessé qu'en 1835 ».(21) Dans les années 1870, une campagne fut lancée pour éradiquer ces usages que les élites jugeaient barbares. ${ }^{(22)}$

Le culte de la force brutale caractérisait donc cette société villageoise. Le militant démocrate-socialiste Pierre Joigneaux, se remémorant, dans une monographie consacrée à sa commune natale, les bagarres qui opposaient dans sa jeunesse les enfants du village, dénonce ce goût populaire du caïd : il explique l'immense popularité dont jouissait, selon lui, l'empereur Napoléon III dans les campagnes. ${ }^{(23)}$ Ceux qui, par leur comportement, s'écartaient de ce modèle de masculinité couraient le risque d'être stigmatisés ou marginalisés. Les instituteurs de la Belle-Époque firent ainsi la douloureuse expérience de l'isolement moral au sein de collectivités villageoises dont ils devenaient paradoxalement l'un des pivots. "Dans la communauté paysanne de la fin du XIXème siècle, dominée par les valeurs viriles et fortement marquée par la division symbolique du travail entre les sexes, le monde des livres tend parfois à se confondre avec le monde des femmes, avec l'“intérieur", et à s'opposer au monde des hommes, de la force physique, de l'“extérieur". " (24) Les écrits autobiographiques des instituteurs de la IIIème République témoignent de cette sorte de honte que ces hommes issus pour la plupart des classes populaires, mais passés par l'École normale et ne travaillant pas de leurs mains, éprouvaient pour un corps sousemployé, fragile, féminisé. ${ }^{(25)}$

\section{Guerres intervillageoises et masculinité}

La participation des jeunes paysans aux rixes ou guerres intervillageoises constituait l'un des moments forts du processus de différenciation des rôles 
sexuels. C'est également dans cette perspective qu'il faut interpréter cette forme de violence, si caractéristique de la société rurale traditionnelle, et dont on a surtout souligné qu'elle contribuait à la reproduction des identités territoriales. L'étude de la correspondance judiciaire et des rapports préfectoraux révèle que ces combats étaient beaucoup plus fréquents et beaucoup plus meurtriers dans les campagnes du Haut-Quercy que dans tout le reste de la France. C'est dans ce cadre géographique que nous nous efforcerons d'observer cette violence spécifiquement juvénile.

Le concept de cycle vindicatoire, emprunté à l'anthropologie, ${ }^{(26)}$ permet de rendre compte de la dynamique des rivalités intercommunales. Les relations qu'entretenaient les habitants de deux localités voisines étaient fondées sur l'alternance de périodes de paix et de périodes de "guerre " (l'expression est utilisée par les populations elles-mêmes), au cours desquelles les contacts et les échanges ordinaires devenaient problématiques, se raréfiaient, et, dans certains cas, s'interrompaient totalement.

Une simple provocation (insulte, bousculade au moment des danses un jour de fête votive) suffisait à déclencher un cycle de violences. Pendant plusieurs semaines, parfois plusieurs mois, les jeunes célibataires des deux communes ennemies s'affrontaient à coups de bâtons et de pierres, profitant de la tenue d'une foire ou d'une fête de village pour régler leurs différends. Au cours de ce cycle, la violence allait généralement croissant, chaque défaite subie impliquant, de la part du camp vaincu, une revanche éclatante. Les mesures adoptées par les autorités pour enrayer cette dynamique vindicatoire s'avéraient peu efficaces, quand elles n'étaient pas dangereuses, une condamnation en justice ne contribuant en général qu’à exciter la colère de ceux qui la subissaient. Seule une réconciliation rituelle pouvait mettre fin à un cycle vindicatoire : après de délicats pourparlers, les représentants des deux partis se rencontraient à la frontière des deux localités, parfois en terrain neutre : l'échange de vin, de menus cadeaux, d'amabilités, les réjouissances collectives venaient marquer le rétablissement de relations pacifiques. Quelques mois ou quelques années plus tard, une nouvelle provocation inaugurait un nouveau cycle de violences.

Ce qui était en jeu, dans les affrontements entre localités voisines par jeunesse interposée, c'était l'honneur du groupe, principal fondement de l'identité villageoise dans une contrée où l'institution communale était particuliè-
(25) Le goût insolite des livres et des choses de l'esprit pouvait être perçu comme la conséquence d'un accident physique. Ibid., p. 100. Jean-Marie Déguignet qui, après une enfance misérable dans les environs de Quimper, apprit seul à lire et à écrire, attribue le développement de ses facultés intellectuelles ainsi que son vif désir de s'instruire à l'accident qui lui avait ouvert le crâne. Mémoires d'un paysan bas-breton, Le Releck-Kerhuon, An Here, 1998, 462 p. Philippe Boutry souligne la dimension virile, machiste, de l'anticléricalisme rural : Prêtres et paroisses au pays du curé d'Ars, Paris, éditions du Cerf, 1986, 506 p. Au XIXème siècle, la jeunesse villageoise était d'ailleurs à la pointe de la contestation du clergé.

(26) Voir en particulier Raymond Verdier, 
La vengeance, tome 1 ,

Études d'ethnologie,

d'histoire et de philosophie

et tome 2, Vengeance et

pouvoirs dans quelques

sociétés extra-occidentales,

Paris, Cujas, 1980,

224 et $196 \mathrm{p}$.

(27) Sur cette dimension des danses traditionnelles : Jean-Marie

Guilcher, «Aspects et problèmes de la danse populaire traditionnelle", Éthnologie française, t. I, $\mathrm{n}^{\circ} 2,1971$, p. 7-48; Jean-Claude Farcy, « Le temps libre au village (1830-1930) ", in Alain Corbin, [dir.], L'avènement des loisirs. I850-1960, Paris, Aubier, 1995, p. 230274 ; Michael R. Marrus, «Modernization and Dancing in Rural France : From "la Bourrée" to "le Fox-Trot" ", in The Wolf and the Lamb, op. cit., p. 141-159.

(28) Les taux d'endogamie élevés s'expliquent tout simplement par rement rudimentaire dans ses structures (la commune ne formait pas ici une communauté agraire). Ces violences n'avaient pas d'autre motif que la défense du point d'honneur collectif. Il n'était pas rare qu'à l'occasion d'une fête patronale, les garçons du village s'exposassent volontairement à subir une offense, par exemple en invitant leurs adversaires à venir s'emparer du « bâton d'honneur » qu'ils avaient confectionné, en l'ornant de rubans, dans les jours ou les semaines précédant l'événement. Ce bâton, brandi au moment des danses, c'est-à-dire lorsque la collectivité communiait dans la liesse, ${ }^{(27)}$ était tout à la fois un emblème de fierté collective et un symbole de virilité. En relevant le défi, en lavant l'affront par la violence, les garçons du village manifestaient l'existence du groupe, soudé dans la conscience d'avoir une réputation à défendre. Pareille conception, toute virile, de l'honneur expliquait la brutalité des relations intergroupales.

On observera encore que la fréquentation des filles fournissait souvent le prétexte aux rivalités entre garçons de communes voisines. Toutefois, contrairement à ce qu'on a fréquemment affirmé, il ne s'agissait en aucun cas d'empêcher les "forains " de venir épouser les filles du village. Les communes françaises n’ont jamais formé des isolats ; ${ }^{(28)}$ la résidence, bien plus que l'appartenance à la commune, constituait l'un des critères de choix du conjoint. En revanche, les conflits intercommunaux provoqués par les fréquentations amoureuses révèlent une conception du groupe territorial qui semble reproduire le modèle familial, en ce sens qu'elle était fondée sur l'opposition de l'interne, domaine des femmes, et de l'externe, domaine des hommes. ${ }^{(29)}$

Si elles étaient fréquemment, et bien involontairement, la cause de ces querelles, les filles n'y prenaient pas une part active. Leur participation n'était jamais qu'indirecte. Cette violence était d'abord une affaire d'hommes, et d'hommes jeunes. La moyenne d'âge des combattants était de 26,9 ans. 86,9\% avaient entre 18 et 35 ans. Seuls les garçons célibataires étaient généralement admis sur le champ de bataille. Il est vrai que, d'une certaine manière, toute la population se trouvait impliquée dans ces violences, qui ne relevaient en aucun cas de la déviance : jamais les combattants n'étaient dénoncés par leurs compatriotes qui, au contraire, s'efforçaient, une fois les poursuites engagées, de les disculper. À l'inverse, il arrivait que des couards qui se dérobaient soient sanctionnés par une assouade ou condamnés à payer une amende. Et on voyait 
souvent des vieillards reprocher aux jeunes générations de ne pas se montrer dignes de leurs ancêtres, qui, eux, savaient se battre.

La participation à ces rixes - qui prenaient des allures de véritables guerres - était une étape importante dans l'intégration de l'adolescent au groupe de jeunesse. Elle fournissait au jeune paysan au sortir de l'enfance l'occasion de faire étalage de sa virilité. Faire bonne figure sur le champ de bataille, c'était savoir se comporter en homme, les principales qualités requises étant la force, l'adresse et le courage.

À compter de la seconde moitié du XIXème siècle, on observe une transformation radicale des formes de la sociabilité juvénile. Cette mutation correspond à la mise en place, notamment sous la IIIème République, d'un réseau d'associations communales spécialisées dans le loisir (sport, musique, lecture...). Tous ces organismes sont contrôlés par des représentants d'une bourgeoisie de village détentrice du pouvoir municipal. Par conséquent, les jeunes paysans qui, dans la société traditionnelle, jouissaient collectivement, une fois sortis du cadre de la maisonnée et de l'exploitation, d'une très large autonomie, sont désormais placés sous la tutelle des adultes. L'essor de cette vie associative s'inscrit dans une vaste entreprise d'acculturation des populations villageoises. Il s'agit en effet pour cette petite élite rurale, qui joue le rôle de relais dans la diffusion des valeurs de la bourgeoisie urbaine, d'éradiquer certaines des manifestations les plus " scandaleuses " de la culture juvénile, telles que la licence, la violence ou les tapages nocturnes. ${ }^{(30)}$ La mobilisation des adhérents, régulièrement convoqués pour des réunions, des exercices ou des concours, désorganise la sociabilité informelle. Et l'obligation faite aux jeunes sociétaires de respecter des statuts et un règlement favorise l'apprentissage de nouvelles normes de conduite.

Cette sociabilité encadrée a beaucoup contribué à la disqualification des violences juvéniles. Elle offrait aux jeunes garçons la possibilité d'exhiber leur virilité dans des formes codifiées : à l'occasion notamment de rencontres sportives. Les vieilles rivalités intercommunales pouvaient désormais s'exprimer pacifiquement à l'occasion de ces compétitions, ou encore dans le cadre de joutes musicales. la faible extension des marchés matrimoniaux.

(29) L'exploration des marges du terroir faisait partie des rites d'initiation des garçons au sortir de l'enfance.

(30) Sur ces questions : Pierre Goujon,

«Association et vie associative dans les campagnes au XIXème siècle : le cas du vignoble de Saôneet-Loire ", Cahiers d'histoire, t. XXVI, 1981, p. 107-150. Christian Thibon a souligné l'importance des arrêtés de police municipale dans la diffusion des valeurs de la civilisation urbaine. Pays de Sault. Les Pyrénées audoises au XIXème siècle. Les villages et l'État, Paris, CNRS, 1988, 278 p. 